\title{
Importance de la déformation interne des roches dans la détermination de la déformation régionale
}

\author{
Importance of the internal rock deformation in the determination \\ of the regional deformation
}

\author{
M. SPECHT, J. DÉRAMOND
}

Laboratoire de Géologie structurale et Tectonophysique"

Rev. Franç. Géotech. n 56, pp. 33-42 (juillet 1991)

\section{Résumé}

L'importance de la déformation interne dans les reconstitutions rétrotectoniques (coupes équilibrées) est discutée. Un exemple pris dans les Pyrénées, intégrant des mesures de déformation, est développé.

\footnotetext{
Abstract

The importance of the internal deformation in the construction of balanced crosssections is discussed. An example, from the tectonic belt of the Pyrenees is exposed.
} 
Pour reconstituer la géométrie des zones en raccourcissement (chaînes de montagnes), il est devenu classique d'utiliser la technique des coupes équilibrées sur une comparaison entre l'état initial (avant déformation) et l'état final, sur une coupe verticale perpendiculaire aux structures.

Dans la plupart des reconstitutions, on admet que la déformation interne continue est négligeable devant la déformation résultant de déplacements discontinus le long des surfaces de failles.

Cette hypothèse sert d'argument aux détracteurs de la méthode des coupes équilibrées pourtant fort utile pour interpréter la géométrie des chaînes de montagne.

Dans les constructions basées sur la technique des coupes équilibrées, il est souvent nécessaire de comparer l'aire d'une surface limitée entre deux repères géologiques linéaires (niveaux stratigraphiques) dont la position finale est connue et la position initiale estimée. Cette aire, en l'absence de toute déformation interne, est la même dans l'état initial et final.

\section{LE PROBLÈME GÉOLOGIQUE}

On se propose, en prenant comme exemple la zone axiale des Pyrénées, de montrer l'importance de la déformation interne.

\subsection{La structure alpine de la zone axiale des Pyrénées : un empilement antiformal d'écailles paléozoïques}

La zone axiale de la chaîne des Pyrénées est constituée par un ensemble d'écailles de matériel paléozoï- que chevauchantes et déplacées vers le Sud lors de l'orogenèse alpine (WILLIAMS et FISCHER, 1984 ; DÉRAMOND et al., 1985 ; SÉGURET et DAIGNIÉRES, 1986) (fig. 1 et 2) empilées les unes sur les autres et constituant donc un empilement antiformal. Cet ensemble d'écailles est limité par (fig. 3) :

- un décollement basal: cette surface structurale recoupe obliquement des séries paléozoïques déjà structurées pendant lorogenèse hercynienne ; ce chevauchement de mur est le plan de subduction (ou de sous-charriage) de l'Ibérie sous l'Europe;

- un décollement de toit qui correspond au sommet des séries paléozoïques, matérialisées par les évaporites du Keuper.

Ces chevauchements, de mur et de toit, se branchent, au Sud, sous les épaisses séries mésozoïques de la partie septentrionale de l'Unité sud pyrénéenne centrale (USPC) (fig. 3) et se propagent dans les zones externes méridionales par des chevauchements et des décollements à travers les séries sédimentaires postpermiennes jusqu'au Bassin de l'Ebre.

Un tel dispositif se rapporte, d'un point de vue géométrique, à un empilement antiformal (BOYER et al., 1982) d'échelle crustale : le raccourcissement relayé par cet empilement antiformal est amorti au front de celui-ci (fig. 3).

Les études de terrain permettent une connaissance relativement bonne de la géométrie du décollement supérieur, mais on connaît peu d'informations sur celle du chevauchement inférieur (plan de subduction de l'Ibérie vers le nord). En accord avec les résultats des profils ECORS (ECORS-Team, 1988), nous avons considéré qu'il s'agissait d'une surface plane pentée vers le Nord

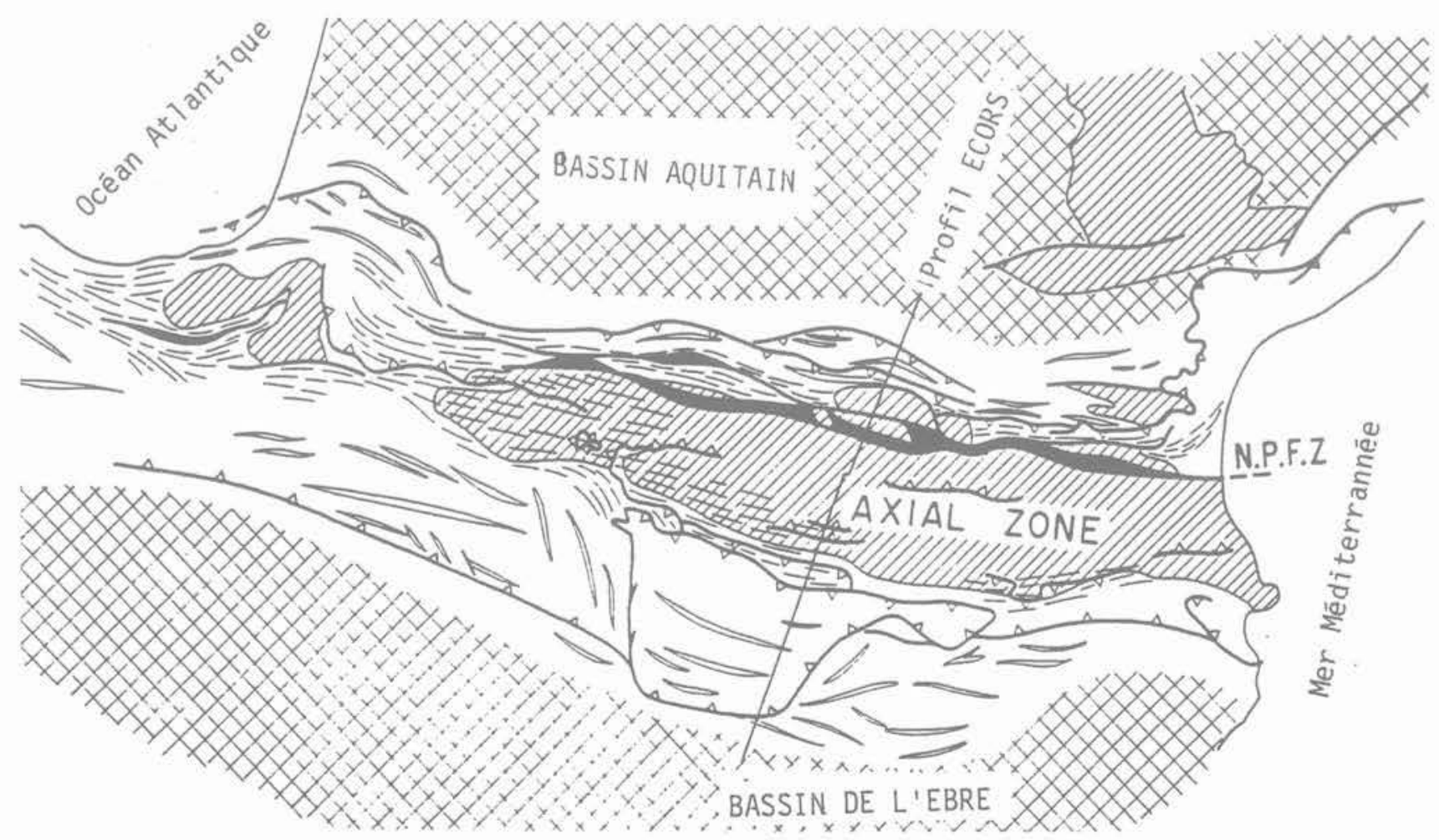

Fig. 1. - Carte de situation du profil ECORS-Pyrénées (d'après ROURE et al., 1989).

Fig. 1. - Location of the ECORS-Pyrenees profile (from ROURE et al., 1989). 


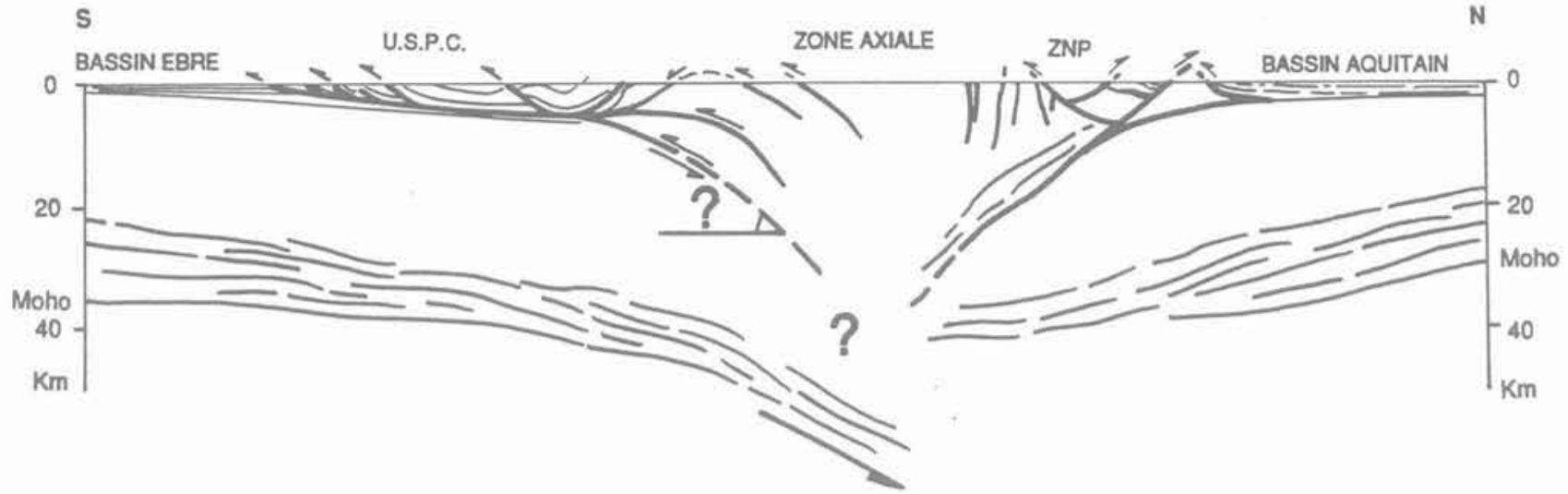

Fig. 2. - Coupe synthétique de la structure de la chaine alpine des Pyrénées le long du profil ECORS (en partie d'après PUIGDEFABREGAS et al., 1988 et ECORS-Team, 1988). Noter que seule la partie méridionale de la zone axiale est structurée en empilement antiformal à vergence sud. L'élévation des massifs nord-pyrénéens et de la partie nord de la zone axiale est liée au jeu des chevauchements nord-pyrénéens.

Fig. 2. - Synthetic cross-section of the alpine tectonic belt of the Pyrenees along the ECORS-profile

(in part from PUIGDEFABREGAS et al., 1988 et ECORS-team, 1988). Note that the southern part of the Axial Zone is structured as an antiformal stack with a southern vergence. The uplift of the North pyrenean massifs in the northern part of the Axial Zone is due to thrusting.

S

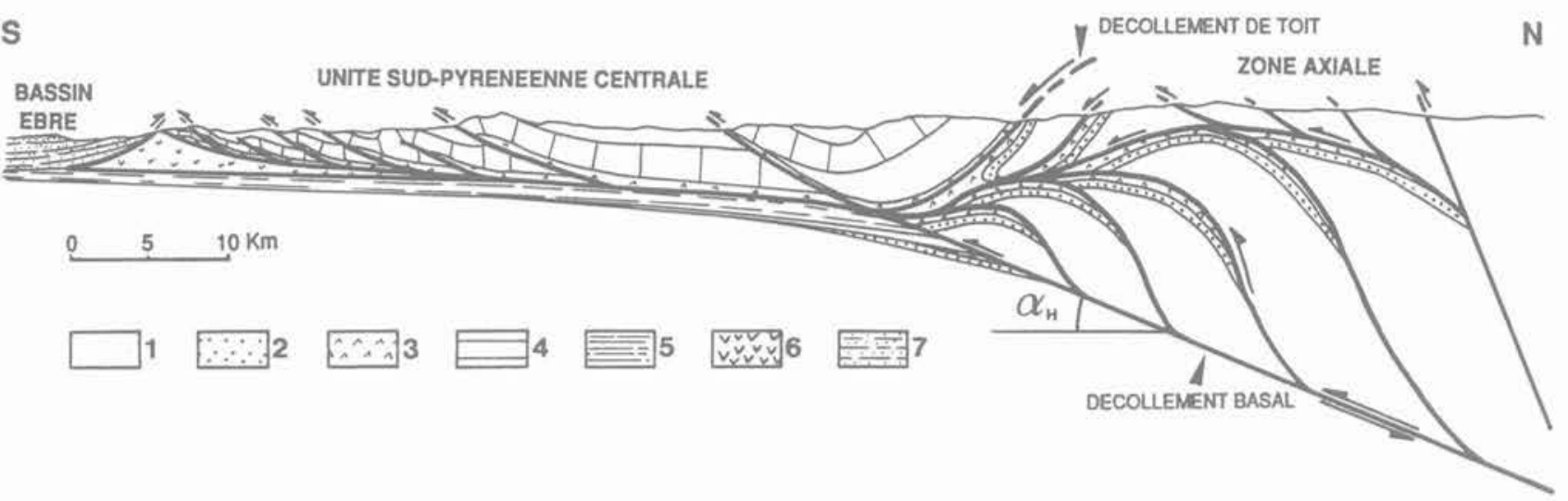

Fig. 3. - Coupe simplifíé du versant sud des Pyrénées. La partie méridionale de la zone axiale est interprétée comme un empilement antiformal d'écailles de paléozoïque dont le décollement basal plonge vers le nord avec un angle $\alpha_{H}$ 1. paléozoïque ; 2. stéphanien et permien (conglomérats, grès et pélites); 3. keuper (évaporites); 4 . mésozoïque (marnes et carbonates) ; 5. tertiaire (faciès marneux); 6. eocène supérieur (évaporites) ; 7. oligocène (marnes et grès).

Fig. 3. - Simplified section of the southern par of the Pyrenees. The antiformal stack is interpreted as a imbricate stack with a sole decollement dipping northward with an $\alpha_{H}$ angle. 1. paleozoic series; 2. stephanian to permian conglomerates and sandstones; 3. triassic evaporites; 4. mesozoï marls and limestones; 5 . tertiary maris; 6 . late eocene evaporites; 7. oligocene marls and sandstones.

(fig. 2). Nous avons tenté d'estimer le pendage dont une méthode a été développée, dérivée de celle dite de la "surface transférée " et basée sur le principe que les bilans de longueur au toit et au mur du Paléozoïque sont réalisés à l'échelle du versant sud des Pyrénées ; autrement dit le raccourcissement exprimé dans la partie méridionale de la zone axiale est intégralement transmis et amorti dans les nappes et séries décollées du versant sud des Pyrénées.

\subsection{Déformation interne dans un empilement antiformal}

Dans un empilement antiformal, la série chevauchée est sous-charriée le long du décollement basal dont le pendage s'accentue progressivement au cours du raccourcissement (fig, 3).

La croissance d'un empilement antiformal s'accompagne du plongement de la partie chevauchée; ce type de structure n'est envisageable qu'à l'échelle des plaques.

La somme des déplacements au sein d'un empilement antiformal est égale à la somme des raccourcissements au front de celui-ci si la déformation interne est négligeable; mais comme dans les chaînes de collision cette dernière l'est rarement, il en sera tenu compte dans la formulation.

Hormis le cas d'une déformation plane (déformation nulle selon la direction principale intermédiaire), la 
déformation interne des roches au cours d'un raccourcissement est anisoaire, dans le plan d'observation, les surfaces et les longueurs des séries impliquées dans les chevauchements sont modifiées. Le calcul du pendage du chevauchement basal de l'empilement antiformal doit tenir compte de la déformation interne.

Lors de la construction d'une coupe, deux aspects de la déformation interne doivent être abordés et quantifiés :

- la variation de surface des séries intégrées dans l'empilement antiformal (dans les zones internes des chaines les déformations sont rarement planes homogènes et donc isoaires) ;

- le raccourcissement homogène des séries où s'amortit la somme des déplacements des écailles composant l'empilement antiformal (bien que difficile à quantifier, le négliger conduirait à sous-estimer le raccourcissement total).

\subsubsection{La variation de surface}

Pour une déformation isovolume, anisoaire dans le plan XZ, la variation de la surface d'une coupe entre état initial $\left(\mathrm{S}_{0}\right)$ et état final $\left(\mathrm{S}_{1}\right)$ est égale à :

$$
\frac{\mathrm{S}_{1}}{\mathrm{~S}_{0}}=\lambda_{\mathrm{X}} \lambda_{\mathrm{Z}}=\frac{1}{\lambda_{\mathrm{Y}}}
$$

On note $\mathrm{C}_{\mathrm{S}}=\lambda_{\mathrm{Y}}$ le " coefficient correcteur de surface » qui permet d'annuler l'effet anisoaire d'une déformation et de retrouver la valeur de $\mathrm{S}_{0}$, nécessaire au calcul du pendage d'un empilement antiformal.
$\mathrm{S}_{1}$ est mesuré sur la coupe de l'état actuel ; $\mathrm{C}_{\mathrm{S}}=\lambda_{\mathrm{Y}}$ est calculé par des méthodes statistiques d'analyse de la déformation à partir de données de terrain (cf. J. DÉRAMOND, 1979, pour une revue de ces méthodes).

\subsubsection{Le raccourcissement homogène parallèle au toit de l'empilement antiformal}

Le raccourcissement homogène parallèle aux couches précède généralement les chevauchements et les plis associés (CASAS et MUNOZ, 1987 ; COOPER et al., 1983 ; GEISER, 1988) ; il peut s'exprimer dans l'état actuel, en notant (fig. 4)) :

$$
\lambda=(1+\epsilon)=\mathrm{L}_{\mathrm{T} 1} / \mathrm{L}_{\mathrm{TO}}
$$

$\mathrm{L}_{\mathrm{TO}}=$ longueur avant raccourcissement homogène (dans les séries où s'amortit le raccourcissement exprimé dans l'empilement antiformal) ;

$\mathrm{L}_{\mathrm{T} 1}=$ longueur après raccourcissement homogène (mesurée depuis l'arrière de l'empilement antiformal jusqu'à la ligne d'extrémité des déplacements) ;

par :

$\mathrm{L}_{\mathrm{T} 0}-\mathrm{L}_{\mathrm{T} 1}=\mathrm{L}_{\mathrm{T} 1} \frac{1-\lambda}{\lambda}=\mathrm{L}_{\mathrm{T} 1} \frac{-\epsilon}{1+\epsilon}$

Le raccourcissement est la somme du raccourcissement homogène au toit de l'empilement antiformal $\left(\mathrm{L}_{\mathrm{T} 0}-\mathrm{L}_{\mathrm{T} 1}\right)$ et du raccourcissement exprimé dans les chevauchements et plis associés ( $\mathrm{L}_{0}-\mathrm{L}_{1}$ ) (fig. 4).

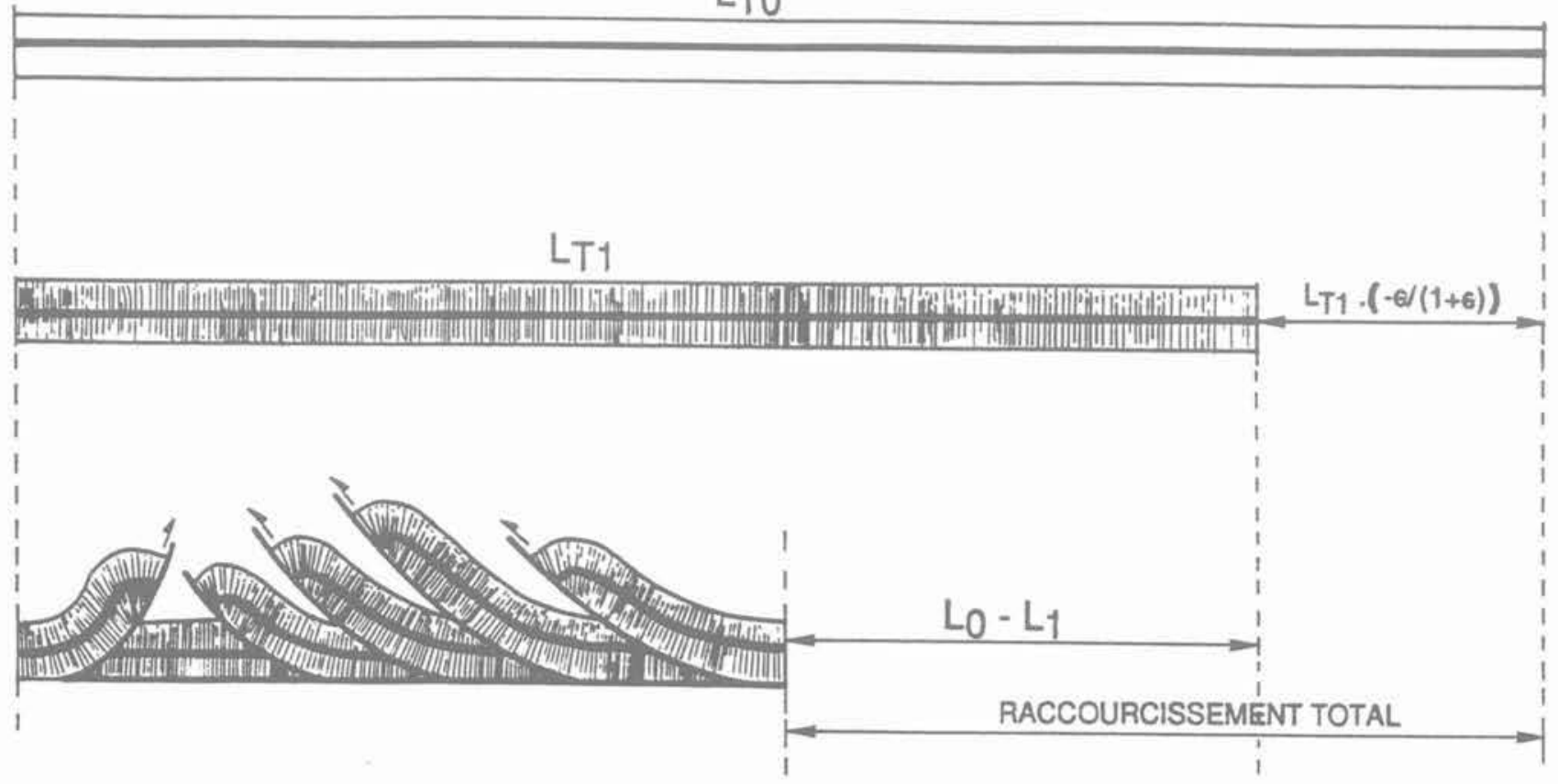

Fig. 4. - Raccourcissement total d'une série sédimentaire : somme du raccourcissement homogène et du raccourcissement par plissement et chevauchement.

Fig. 4. - Total shortening of a sedimentary pile: sum of the homogeneous shortening and of the shortening due to folding and thrusting. 


\subsection{Pendage du chevauchement basal d'un empilement antiformal}

Le pendage du chevauchement basal d'un empilement antiformal s'accentue au cours de la déformation.

La surface en coupe de l'empilement antiformal est égale :

- avant raccourcissement (fig. 6), à :

$$
S_{0}=\frac{T_{0}^{2}-\operatorname{tg} \alpha_{0}}{2}
$$

- après déformation, à :

$$
\mathrm{S}_{1}=\mathrm{S}+\frac{\mathrm{T}_{1}^{2} \cdot \operatorname{tg} \alpha}{2}
$$

avec :

$\mathrm{T}_{0}=$ longueur initiale du toit de l'empilement antiformal ;

$\mathrm{T}_{1}=$ longueur finale de l'empilement antiformal ;

$\alpha=$ pendage actuel du chevauchement;

$\mathrm{S}=$ surface transférée (au-dessus de la position initiale du décollement de toit de l'empilement antiformal).

Si la déformation interne est négligeable (fig. 5), alors $\mathrm{C}_{\mathrm{S}}=\grave{\mathrm{y}}=1$, l'angle $\alpha$ est donné par :

$$
\left.\operatorname{tg} \alpha=\frac{2 \mathrm{~S}}{\mathrm{~T}_{1}\left(\mathrm{~L}_{0}-\mathrm{L}_{1}\right)} \text { (fig. } 5 \text { et } 6\right)
$$

En tenant compte de la déformation interne (fig. 6), on peut écrire:

$$
\mathrm{T}_{0} \cdot \mathrm{T}_{1} \operatorname{tg} \alpha=\left(2 \mathrm{~S}+\mathrm{T}_{1}^{2} \operatorname{tg}_{\alpha}\right) \cdot \mathrm{C}_{\mathrm{S}}
$$

Le pendage du décollement basal de l'empilement antiformal après la déformation est donné par:

$$
\operatorname{tg} \alpha=\frac{2 S C_{S}}{T_{1}\left(T_{0}-T_{1} C_{S}\right)}
$$

avec: $\mathrm{T}_{0}=\mathrm{T}_{1}+\left(\mathrm{L}_{0}-\mathrm{L}_{1}\right)+\mathrm{L}_{\mathrm{T} 1}(-\epsilon /(1+\epsilon))$ où :

$\mathrm{L}_{\mathrm{T} 1}=$ longueur sur laquelle le raccourcissement exprimé dans l'empilement antiformal est amorti (mesuré par curvimétrie sur un banc repère situé au toit de l'empilement antiformal) ;

$\mathrm{L}_{\mathrm{T} O}-\mathrm{L}_{\mathrm{T} 1}=\mathrm{L}_{\mathrm{T} 1}(-\epsilon /(1+\epsilon))$ : raccourcissement homogène parallèle aux couches;

avec :

$\lambda=1+\epsilon=$ extension linéaire mesurée parallèlement aux couches dans le plan de coupe;

$\mathrm{T}_{0}-\mathrm{T}_{1}=$ raccourcissement total;

$\mathrm{L}_{0}-\mathrm{L}_{1}=$ raccourcissement associé aux chevauchement (mesuré au toit et au front de l'empilement antiformal).

\section{LES MESURES}

Les mesures effectuées dans la zone axiale (région de Gavarnie) sont résumées dans le tableau ci-après.

Ces mesures ont été effectuées sur divers marqueurs géologiques. XY est le plan de schistosité ; $\mathrm{X}$ la direction d'extension maximale; $e_{1}, e_{2}, e_{3}$ sont les déformations principales logarithmiques.

\section{RÉSULTATS : PENDAGE DU DÉCOLLEMENT BASAL DU PLAN DE SUBDUCTION IBÉRIE-EUROPE}

Le calcul de $\alpha \mathrm{H}$ (pendage du décollement basal du plan de subduction après déformation) nécessite lestimation des différents paramètres $\left(\mathrm{L}_{0}-\mathrm{L}_{1}, \mathrm{~T}_{1}, \mathrm{~S}\right)$;

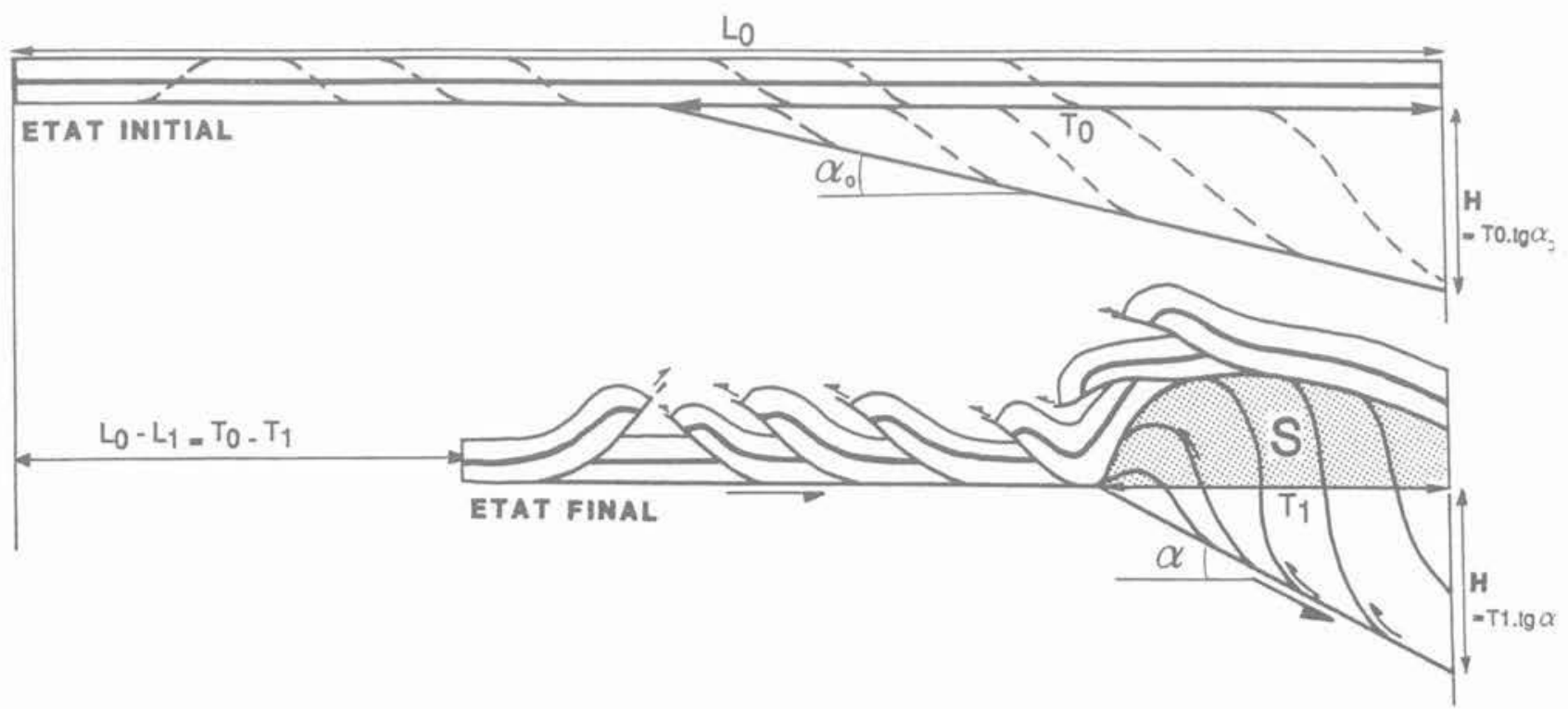

Fig. 5. - Empilement antiformal: paramètres nécessaires au calcul de $\alpha$ et $\alpha_{0}$. Cas d'une déformation isoaire. Fig. 5. - Antiformal stack: parameters of $\alpha$ and $\alpha_{0}$ calculation. Equal area deformation. 


\begin{tabular}{|c|c|c|c|c|c|c|}
\hline \multirow{2}{*}{ Age du terrain } & \multirow{2}{*}{ Marqueurs } & \multicolumn{2}{|c|}{ Orientations } & \multicolumn{3}{|c|}{ Déformations principales } \\
\hline & & $X Y$ & $\mathrm{x}$ & $e_{1}$ & $e_{2}$ & $e_{3}$ \\
\hline \multirow{7}{*}{ Carbonifère } & \multirow{7}{*}{ radiolaires } & $115 \times 65$ & $20 N 65$ & 0,35 & $-0,09$ & $-0,26$ \\
\hline & & $110 \times 70$ & $180 \times 70$ & 0,32 & $-0,09$ & $-0,23$ \\
\hline & & $100 \times 70$ & $180 \times 70$ & 0,34 & $-0,12$ & $-0,22$ \\
\hline & & $130 \times 70$ & 010 N 65 & 0,32 & $-0,04$ & $-0,28$ \\
\hline & & $125 \times 70$ & $015 \times 75$ & 0,33 & $-0,11$ & $-0,22$ \\
\hline & & $100 \mathrm{~N} 60$ & $170 \mathrm{~N} 60$ & 0,37 & $-0,13$ & $-0,24$ \\
\hline & & $130 \mathrm{~N} 60$ & $170 \mathrm{~N} 85$ & 041 & $-0,10$ & $-0,31$ \\
\hline Silurien & taches pyriteuses & $120 \times 30$ & $020 N 35$ & 1,79 & $-0,21$ & $-1,58$ \\
\hline \multirow{3}{*}{ Dévonien inf. \& moyen } & \multirow{3}{*}{ taches limoniteuses } & HORIZ. & 180/HOR & 1,23 & 0,37 & $-1,61$ \\
\hline & & 085 N 35 & 095/HOR & 1,38 & $-0,36$ & $-1,03$ \\
\hline & & 090 N 20 & 095/HOR & 0,74 & 0,32 & $-1,06$ \\
\hline \multirow{3}{*}{ Dévonien inférieur } & \multirow{2}{*}{ "pellets » } & HORIZ. & 015/HOR & 0,94 & 0,15 & $-1,09$ \\
\hline & & $090 \times 15$ & $020 N 15$ & 0,92 & 0,25 & $-1,17$ \\
\hline & taches limoniteuses & HORIZ. & 030/HOR & 1,34 & 0,60 & $-1,94$ \\
\hline \multirow{2}{*}{ Carbonifère } & \multirow{2}{*}{ goniatites (2) } & $085 \times 15$ & $020 \times 15$ & 0,44 & 0,03 & $-0,47$ \\
\hline & & $085 \times 20$ & $010 \times 10$ & 0,39 & 0,03 & $-0,42$ \\
\hline Dévonien sup. & goniatites & HORIZ. & O9O/HOR & 0,32 & $-0,12$ & $-0,20$ \\
\hline Crétacé & quartz (3) & 070 N 10 & $170 \times 10$ & 0,30 & $-0,09$ & $-0,21$ \\
\hline Trias & galets (4) & $080 \times 10$ & $170 \mathrm{~N} 20$ & 0,56 & $-0,16$ & $-0,40$ \\
\hline \multirow{2}{*}{ Crétacé } & galets (boudinage) (5) & 110 N 30 & $020 \times 35$ & 0,53 & 0,39 & $-0,93$ \\
\hline & galets & $090 \times 15$ & $175 \mathrm{~N} 05$ & 0,52 & 0,42 & $-0,94$ \\
\hline Crétacé & galets (boudinage) & & & 0,51 & 0,44 & 0,95 \\
\hline Trias & galets & HORIZ. & O90 HOR & 0,97 & $-0,20$ & 0,76 \\
\hline Crétacé & galets (boudinage) & HORIZ. & 015 HOR & 0,55 & 0,13 & 0,68 \\
\hline \multirow{2}{*}{ Trias } & \multirow{2}{*}{ galets } & $075 \times 10$ & $025 \times 20$ & 0,45 & 0,34 & $-0,79$ \\
\hline & & $070 \times 15$ & $015 \times 15$ & 0,44 & 0,25 & $-0,68$ \\
\hline Crétacé cénomanien & dragées quartz & $085 \times 15$ & $180 \times 10$ & 0,60 & 0,21 & $-0,81$ \\
\hline \multirow{2}{*}{ Santonien } & \multirow{2}{*}{ foraminifères + "pellets $»$} & HORIZ. & 010 HOR & 0,56 & 0,15 & $-0,71$ \\
\hline & & $090 \times 10$ & $020 \times 10$ & 0,16 & 0,04 & $-0,20$ \\
\hline \multirow{3}{*}{ Campano-Maastrichtien } & \multirow{3}{*}{ foraminifères } & $100 \times 35$ & O80 HOR & 0,42 & 0,06 & $-0,48$ \\
\hline & & $110 \times 40$ & 080 E 20 & 0,39 & 0,07 & $-0,46$ \\
\hline & & $105 \times 40$ & O90 HOR & 0,46 & 0,07 & $-0,52$ \\
\hline
\end{tabular}




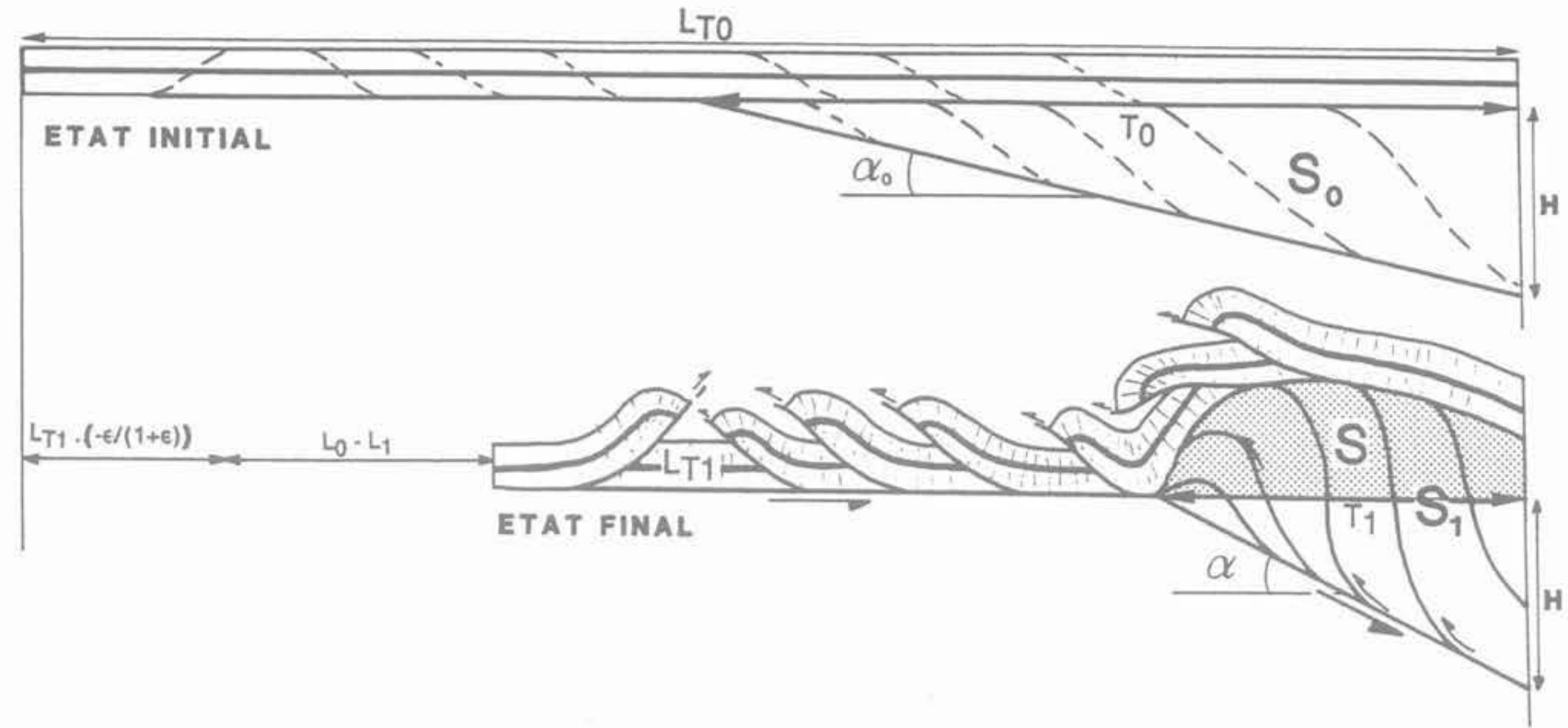

Fig. 6. - Empilement antiformal : paramètres nécessaires au calcul de $\alpha$ et $\alpha_{0}$. Cas d'une déformation anisoaire et anisolinéaire. Fig. 6. - Antiformal stack: parameters of $\alpha$ and $\alpha_{O}$ calculation. Non equal area deformation.

c'est sur S que l'incertitude est la plus forte car sa détermination sur la coupe de l'état actuel nécessite des approximations géologiques dont certaines peuvent être sujettes à caution (par exemple sur la position du toit du Paléozoïque avant et après déformation).

\subsection{Quantification de la déformation interne (des séries paléozoïques au cours de la structuration de la zone axiale en empilement antiformal)}

La quantification de la déformation alpine de la zone axiale des Pyrénées pose deux problèmes, celui des marqueurs et celui des séquences de la déformation.

\subsubsection{Les marqueurs de la déformation}

Les calculs de la déformation des roches s'effectuent par traitement statistique de la géométrie de marqueurs ponctuels qui nécessite un grand nombre de mesures ( 250 à 300 mesures par station), nombre rarement atteint dans la zone axiale des Pyrénées. De plus, dans ces terrains paléozoïques, les marqueurs (goniatites par exemple) enregistrent les effets de deux déformations successives (hercynienne et alpine), ce qui rend délicate la mesure de la déformation alpine.

\subsubsection{La superposition des déformations}

La déformation finie résulte de la superposition d'une déformation hercynienne et d'une déformation alpine (chacune pouvant correspondre à la superposition de plusieurs phases). Faire la part de la déformation alpine n'est possible qu'en de rares points de la zone axiale. Ce seul travail important permettant de caractériser l'ellipsoïde de la déformation alpine, à partir de l'étude des séries paléozoïques, a été effectué par DÉRAMOND (1979) au cours d'une étude sur les mécanismes de structuration de la nappe alpine de Gavarnie (Hautes-Pyrénées, bordure sud de la zone axiale). La nappe de Gavarnie, constituée de séries paléozoïques, est la partie frontale de l'un des « horses " constitutifs de la zone axiale des Pyrénées (PARISH, 1984 ; GRAHAM et al., 1987).

La déformation totale des séries paléozoïques est isovolume mais non plane (DÉRAMOND, 1979) : l'extension est-ouest (perpendiculaire au plan de coupe) n'est pas nulle: l'extension logarithmique globale (hercynienne et alpine) fluctue de - 0,10 à +1.38 .

Les résultats (DÉRAMOND et al., 1976) sur la déformation hercynienne (assez constants dans tout le secteur de Gavarnie) indiquent une extension logarithmique horizontale $e_{\mathrm{Ew}}$ de l'ordre de 0.1 soit $10 \%$. L'extension logarithmique alpine $\left(e_{\mathrm{EW}}\right)_{\mathrm{A}}$ varie alors de - 0,20 à $+1,28$. Sa valeur moyenne est de $\left(e_{E W}\right)_{A}=0,37$ soit $45 \%$.

La déformation interne, contemporaine de la structuration de l'empilement antiformal dans la région de Gavarnie, n'est donc pas isoaire dans le plan de coupe N-S. La variation de surface correspond le plus souvent à une diminution mais aussi, parfois, à une augmentation. Une diminution de surface dans le plan $\mathrm{XZ}$ (vertical, N-S) se conçoit bien. Une augmentation de surface est plus problématique. Peut-être n'est-elle qu'apparente: résultant par exemple de variations spatiales (diminution locale de l'extension hercynienne dans la direction $\mathrm{EW}$ que nous avons supposée constante).

\section{Variation de surface dans le plan de coupe}

En général, les coupes à travers les chaînes de montagnes sont construites dans le plan XZ de la déformation supposée plane $\left(\lambda_{Y}=0\right)$. Cette déformation 
peut être néanmoins anisoaire dans le plan XZ si elle est anisovolume (HOSSAK, 1979).

Dans le secteur de Gavarnie, comme déjà précisé, la déformation est isovolume :

$$
\begin{array}{ll}
\text { soit } & \lambda_{1} \lambda_{2} \lambda_{3}=1 \\
\text { ou : } & e_{1}+e_{2}+e_{3}=0
\end{array}
$$

et EW est une direction principale.

La connaissance de l'extension linéaire $\lambda_{E W}$ fournit la variation de surface dans le plan de coupe N-S égale d'après (1) à :

$$
\frac{\mathrm{S}_{1}}{\mathrm{~S}_{0}}=\frac{1}{\lambda_{\mathrm{EW}}}
$$

L'extension $\lambda_{E W}$ dans le secteur de Gavarnie a été calculée à partir des données (DÉRAMOND, 1979) qui portent sur environ 2500 mesures d'orientation des axes et des rapports axiaux de marqueurs ellip- soïdaux (fig. 7). Ces mesures (effectuées dans les terrains paléozoïques de la partie centrale de la nappe) indiquent une valeur $e_{\mathrm{EW}}=0,47$, soit $\lambda_{\mathrm{EW}}=1,6$. L'extension logarithmique hercynienne étant estimée à 0,1 (DÉRAMOND et RAMBACH, 1979) soit $\left(e_{E W}\right)_{H}=0,1$ ou $\left(\lambda_{E W}\right)_{H}=1,1$, l'extension logarithmique alpine serait donc de $\left(e_{\mathrm{EW}}\right)_{\mathrm{A}}=0,37$ ou $\left(\lambda_{\mathrm{EW}}\right) \mathrm{A}=1,45$ soit $45 \%$.

La variation de surface dans le plan NS dans le secteur de Gavarnie, pendant la croissance de l'empilement antiformal, est alors de :

$$
\frac{S_{1}}{S_{0}}=\frac{1}{\left(\lambda_{E W}\right)_{A}}=0,69 \text { soit } \frac{\Delta S}{S_{0}}=-31 \%
$$

Remarque : Ce calcul dit de la * surface non transférée " (diminution de la surface dans le plan de coupe) utilise la valeur ( $\mathrm{EW}=45 \%$ qui parait très élevée en comparaison de celles mesurées dans d'autres chaî-

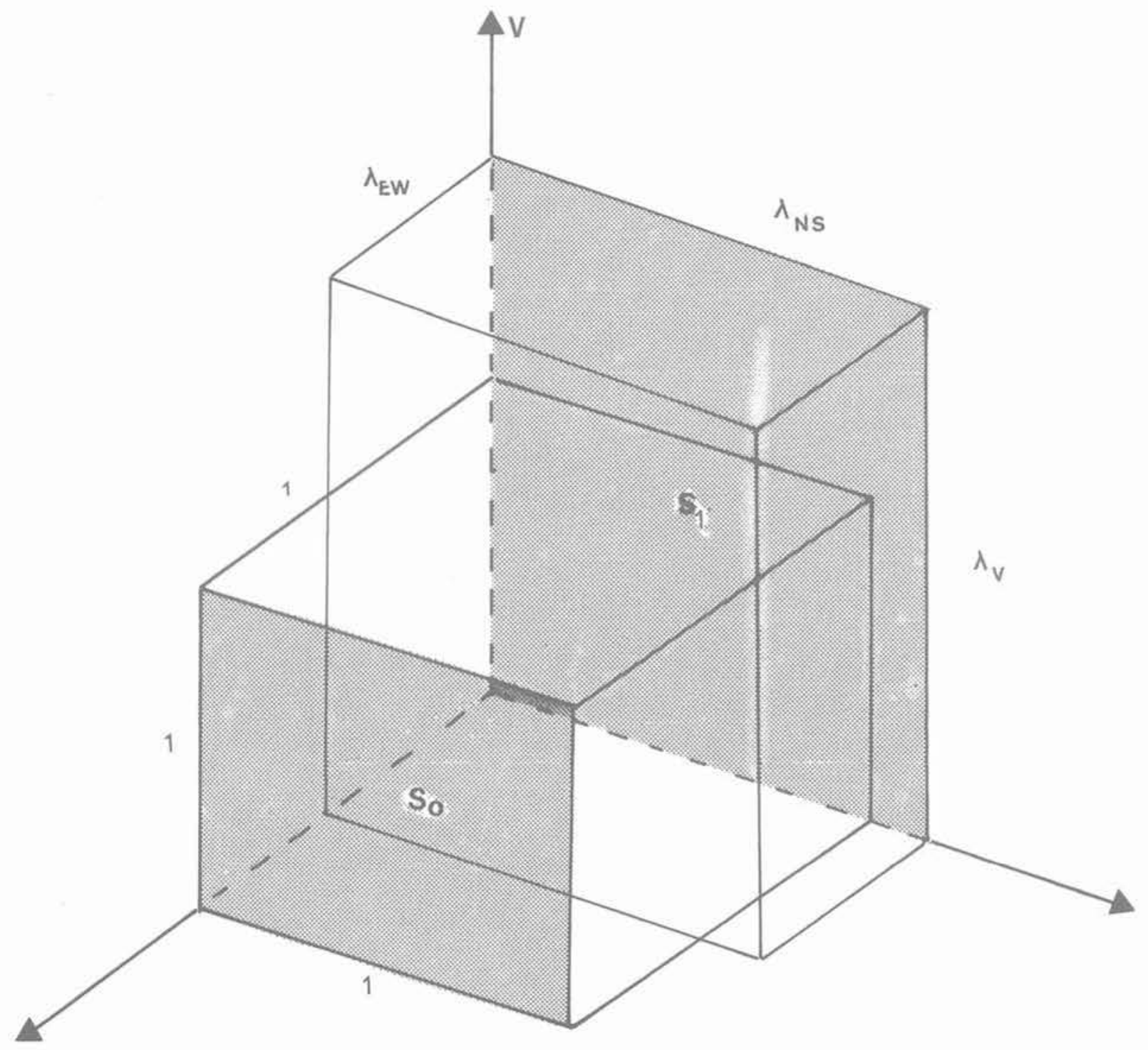

Fig. 7. - Variation de surface dans un plan de coupe, plan principal de la déformation. Fig. 7. - Surface variation in the section plane, principal plane of strain. 
nes de montagne. Ainsi HOSSACK (1979), dans une rapide compilation, ne cite jamais de valeurs supérieures à $15 \%$. De plus, une extension aussi forte est bien sûr difficilement envisageable à l'échelle de la chaîne. Ainsi (cf. paragraphe suivant), la valeur $31 \%$ n'a de signification qu'à l'échelle de la nappe ; elle n'est représentative que de ce domaine et ne peut, en toute rigueur, être étendue à l'ensemble de la zone axiale.

\subsection{Application à la subduction de l'Ibérie}

Certains paramètres $\left(S, T_{0}, T_{1}\right)$, nécessaires au calcul de la position initiale et actuelle du plan de subduction de l'Ibérie, sont connus avec une relativement bonne précision :

$\mathrm{S}=730 \mathrm{~km}^{2} ; \mathrm{T}_{0}-\mathrm{T}_{1}=100 \mathrm{~km} ; \mathrm{T}_{1}=50 \mathrm{~km}$.

En revanche (cf. paragraphe 1 ), $\mathrm{C}_{\mathrm{S}}$ est difficile à déterminer de façon exacte ; nous proposons donc de calculer les pendages pour trois valeurs de $\mathrm{C}_{\mathrm{S}}$ dont deux sont des valeurs limites (tableau 1):

$-\mathrm{C}_{\mathrm{S}}=1,45:$ valeur déduite des mesures à Gavarnie; (la valeur de $\alpha$ est surestimée) :

$\mathrm{C}_{\mathrm{S}}=1$ : la déformation alpine est supposée plane (ce qui revient à sous-estimer la valeur de $\alpha$ );

$-\mathrm{C}_{\mathrm{S}}=1,18$ : valeur intermédiaire (moyenne harmonique des deux valeurs limites précédentes) cohérente avec celles calculées dans d'autres chaînes de collision (HOSSACK, 1979).

\section{Sur la coupe de l'état actuel (fig. 7).}

Pour la partie méridionale de la zone axiale des Pyrénées, $\alpha$ est obtenu à partir de (4b).

L'application numérique montre que l'angle $\alpha$ entre le plan de subduction après raccourcissement et le toit du paléozoïque avant déformation est compris entre $16^{\circ}\left(C_{S}=1\right)$ et $28^{\circ}\left(C_{S}=1,45\right)$ et que sa valeur se situe probablement autour de $21^{\circ}\left(\mathrm{C}_{\mathrm{S}}=1,18\right)$.

Le pendage actuel du plan de subduction est donc probablement voisin de:

$$
\alpha_{H}=\alpha+9^{\circ}=30^{\circ} \mathrm{N}
$$

Tableau 1. - Valeurs de $\alpha_{r} \alpha_{H}, \alpha_{O}, \alpha_{O H}$ pour différentes valeurs de $C_{S}$ avec $S=730 \mathrm{~km}^{2}, T_{1}=50 \mathrm{~km}$ et $T_{0}-T_{1}=150 \mathrm{~km}$.

Table 1. $-\alpha, \alpha_{H}, \alpha_{O}, \alpha_{O H}$ values for different $C_{S}$ with $S=730 \mathrm{~km}^{2}, T_{1}=50 \mathrm{~km}$ and $T_{0}-T_{T} M=150 \mathrm{~km}$.

\begin{tabular}{|c|c|c|c|}
\hline Cs & 1,43 & 1,18 & 1 \\
\hline$\alpha$ & $28^{\circ}$ & $21^{\circ}$ & $16^{\circ}$ \\
\hline$\alpha_{H}$ & $37^{\circ}$ & $30^{\circ}$ & $25^{\circ}$ \\
\hline$\alpha_{\mathrm{O}}$ & $10^{\circ}$ & $7,5^{\circ}$ & $5,5^{\circ}$ \\
\hline$\alpha_{\mathrm{OH}}$ & $15^{\circ}$ & $12,5^{\circ}$ & $10,5^{\circ}$ \\
\hline
\end{tabular}

(la valeur $9^{\circ}$ correspond à langle entre le toit du paléozoïque et l'horizontale dans l'état initial).

Sur la coupe de l'état restauré (fig. 8).

L'angle $\alpha_{0}$ entre l'inclinaison du futur plan de subduction et le toit du paléozoïque avant déformation est donné par la formule (4a).

Pour $C_{S}$ variant entre les valeurs limites 1 et 1,43 , $\alpha_{0}$ est compris entre $5,5^{\circ}\left(\mathrm{C}_{\mathrm{S}}=1\right)$ et $10^{\circ}\left(\mathrm{C}_{\mathrm{S}}=\right.$ $1,43)$, sa valeur étant probablement voisine de $7,5^{\circ}$ $\left(C_{S}=1,18\right)$. Le pendage du plan de subduction dans l'état restauré (Garumnien mis à l'horizontale) est donc de l'ordre de:

$$
\left(\alpha_{0}\right)_{H}=\alpha_{0}+5^{\circ}=12^{\circ} \mathrm{N}
$$

(la valeur $5^{\circ}$ correspond à langle entre le Garumnien et l'horizontale).

Discussion et conclusion : dans le but de contraindre la construction d'une coupe équilibrée d'échelle crustale le long du profil ECORS-Pyrénées, nous avons tenté de reconstituer la géométrie du plan de subduction de l'Ibérie vers le nord. Pour cela a été développée une méthode originale basée sur la méthode de la surface transférée dans laquelle intervient la déformation interne. A la suite de son application, deux points doivent être soulignés:

1. la prise en compte de la déformation interne à l'échelle d'une chaîne de collision nécessite un très grand nombre de mesures (plusieurs milliers) souvent complexes à traiter ; de plus cette démarche, très longue, ne peut être réalisée que dans la partie superficielle d'un tel édifice structural, et seules des extrapolations, évidemment discutables, permettent de proposer des valeurs de déformation dans les zones profondes d'une chaîne;

2. le calcul du pendage du plan de subduction de l'Ibérie vers le nord montre que les résultats sont peu différents, que l'on tienne compte ou non de la déformation interne : la différence est de $5^{\circ}$ pour la valeur du pendage actuel du plan de subduction $\left(30^{\circ}\right.$ $\left(C_{S}=1,18\right)$ contre $\left.25^{\circ}\left(C_{S}=1\right)\right)$ et seulement de $2^{\circ}$ pour celle du pendage de ce plan avant raccourcissement $\left(12,5^{\circ}\right.$ contre $\left.10,5^{\circ}\left(C_{S}=1\right)\right)$.

Ainsi, dans le cas présent, il apparaît que l'extrapolation de mesures de déformation interne à l'échelle d'une chaîne (opération toujours délicate surtout dans le cas de la superposition de déformations) n'est pas nécessaire. Une coupe équilibrée pourrait donc être construite sans tenir compte de cette déformation et les résultats pourraient paraître cohérents. Cependant, le problème de la mesure de la déformation interne dans l'ensemble de la chaîne et son intégration dans la construction des coupes reste posé, car ici on a utilisé des mesures ponctuelles extrapolées à une coupe de plusieurs centaines de kilomètres de long. Une meilleure précision dans la détermination de la déformation régionale à partir des coupes équilibrées suppose soit la multiplication des mesures de déformation interne tout le long de la coupe, soit la mise au point de méthodes plus performantes d'intégration de données ponctuelles à l'échelle d'une coupe. 

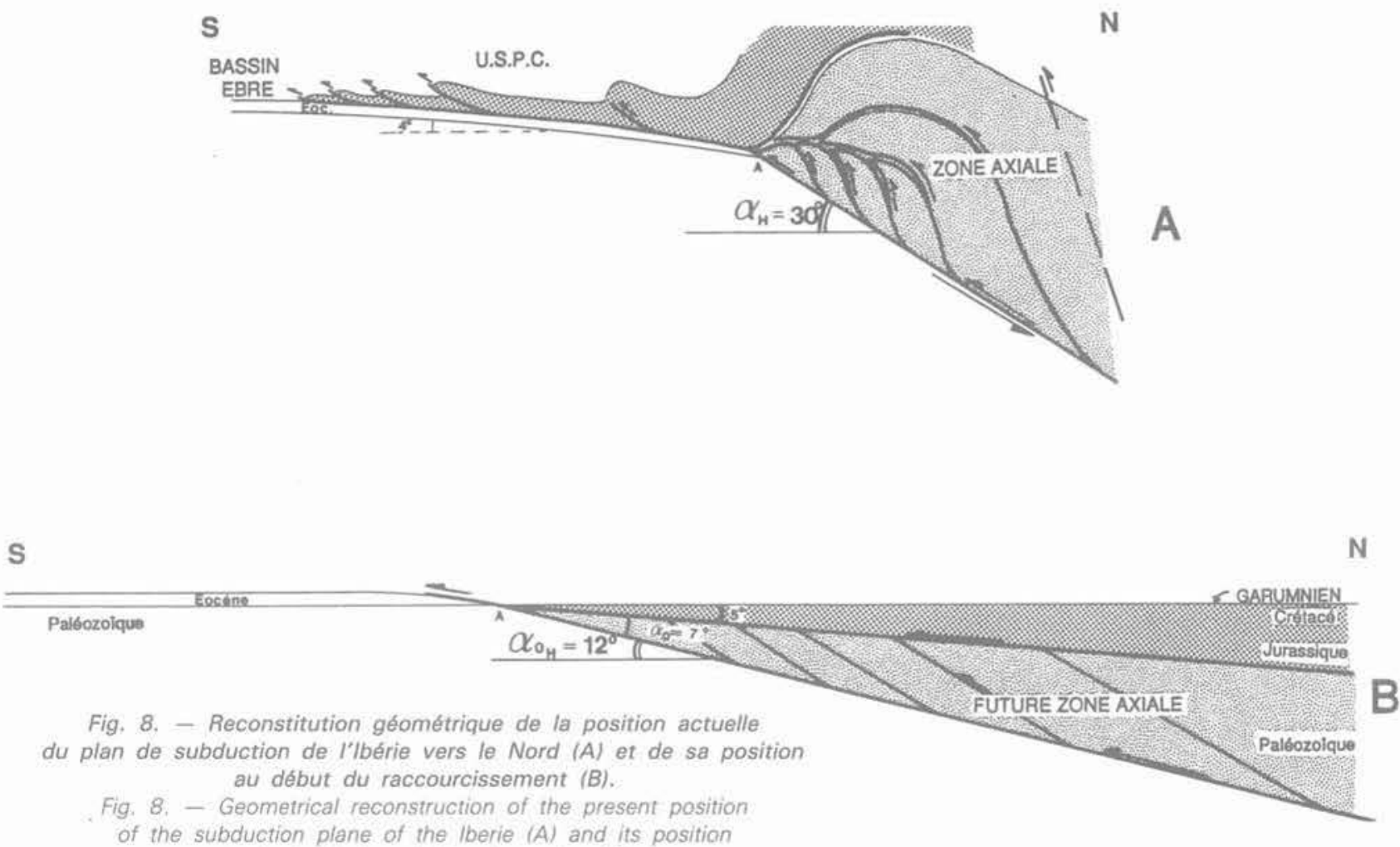
of the subduction plane of the Iberie (A) and its position at the beginning of the shortening $(B)$.

\section{BIBLIOGRAPHIE}

BOYER S.E., ELLIOTT D. (1982), The geometry of thrust systems. A.A.P.G. Bull., vol. 66, n० 9, p. 1196-1230.

CASAS J.M., MUNOZ J.A. (1987), Sequences of mesostructures related to the development of Alpine thrusts in the Eastern Pyrenees. Tectonophysics, vol. $135, n^{\circ} 5$, p. 67-75.

COOPER M.A., GARTON M.R., HOSSACK J.R. (1983), The origin of the Basse-Normandie duplex, Boulonnais, France. J. Struct. Geol., vol. $5, n^{\circ} 2$, p. 139-152.

DÉRAMOND J, (1979), Déformations et déplacement des nappes : exemple de la nappe de Gavarnie (Pyrénées centrales). Thèse Doct. Etat, Toulouse, 409 p.

DÉRAMOND J., GRAHAM R.H., HOSSACK J.R., BABY P., CROUZET G. (1985), Nouveau modèle de la chaîne des Pyrénées. CR Acad. Sc. Paris, t. 301, sér. II, n 16 , p. 1213-1216.

DÉRAMOND J., BABY P., CROUZET G, SPECHT M. (1989), Influence de l'anisotropie sur la géométrie des chevauchements. Exemples de la zone nord-pyrénéenne. CR Acad. Sci. Paris, t 308, sér. II, p. 1481-1484.

ECORS-Pyrénées Team (1988), Deep reflection seismic survey across an entire orogenic belt : the ECORS-Pyrenees profile. Nature, vol. 331, p. 508-511.

GEISER P.A. (1988), Mechanisms of thrust propagation: some examples and implications for the analysis of overthrust terranes. J. Struct. Geol., vol. $10, n^{\circ} 8$, p. $829-845$.

GRAHAM R., HOSSACK J.R., DÉRAMOND J., SOULA J.C. (1987), Géométrie des surfaces de chevauchement. Bull. Soc. géol. France, (8), t. III, $\mathrm{n}^{\circ} 1$, p. 169-181.

HOSSACK J.R. (1979), The use of balanced cross sections in the calculation of orogenic contractiona review. Journ. Geol. Soc., London, vol. 136. p. $115-166$.

PARISH M. (1984), A structural interpretation of a section of the Gavarnie nappe and its implications for Pyrenean geology. J. Struct. Geol., vol. 6 , $n^{\circ} 3$, p. 247-255.

RAMSAY J.G. (1967), Folding and fracturing of rocks. Ed. Mac Graw Hill., 568 p.

SÉGURET M., DAIGNIĖRES M. (1986), Crustal scale balanced cross-sections of the Pyrenees ; discussion. Tectonophysics, vol. 129, $\mathrm{n}^{\circ} 1-4$, p. 303-318.

SOUQUET P., DÉRAMOND J. (1989), Séquence de chevauchements et séquences de dépôt dans un bassin d'avant-fosse. Exemple du sillon crétacé du versant sud des Pyrénées (Espagne). CR Acad. Sc. Paris, t. 309. sér. II, p. 137-144.

SPECHT M. (1989), Tectonique de chevauchement le long du profil ECORS-Pyrénées: un modèle d'évolution de prisme d'accrétion continental. Thèse Univ. de Brest et Toulouse, $353 \mathrm{p}$.

WILLIAMS F.S., FISCHER M.W. (1984), A balanced section across the Pyrenean orogenic belt. Tectonics, vol. 3, p. 773-780. 